\title{
Histological Study on the Effect of Electrolyzed Reduced Water-Bathing on UVB Radiation-Induced Skin Injury in Hairless Mice
}

\author{
Kyung Su Yoon, ${ }^{a}$ Xue Zhu Huang, ${ }^{d}$ Yang Suk Yoon,${ }^{a, b}$ Soo-Ki KIm, ${ }^{c}$ Soon Bong Song, ${ }^{a}$ \\ Byung Soo ChAnG, ${ }^{e}$ Dong Heui KIM, ${ }^{*, a}$ and Kyu Jae LeE ${ }^{*, a, b}$ \\ ${ }^{a}$ Department of Environmental Medical Biology, Wonju College of Medicine, Yonsei University; ${ }^{c}$ Department of \\ Microbiology, Wonju College of Medicine, Yonsei University; Wonju, Gangwon 220-701, South Korea: ${ }^{b}$ Institute for \\ Poverty Alleviation and International Development, Yonsei University Wonju Campus; Wonju, Gangwon 220-710, South \\ Korea: ${ }^{d}$ Department of Anesthesiology, Yanbian University Hospital, Yanbian University; Yanji, Jilin 133000, China: and \\ ${ }^{e}$ Department of Cosmetology, Hanseo University; Seosan, Chungnam 356-706, South Korea. \\ Received March 28, 2011; accepted August 22, 2011; published online September 1, 2011
}

Electrolyzed reduced water (ERW), functional water, has various beneficial effects via antioxidant mechanism in vivo and in vitro. However there is no study about beneficial effects of ERW bathing. This study aimed to determine the effect of ERW bathing on the UVB-induced skin injury in hairless mice. For this purpose, mice were irradiated with UVB to cause skin injury, followed by individually taken a bath in ERW (ERW-bathing) and tap water (TW-bathing) for $21 \mathrm{~d}$. We examined cytokines profile in acute period, and histological and ultrastructural observation of skin in chronic period. We found that UVB-mediated skin injury of ERW-bathing group was significantly low compared to TW control group in the early stage of experiment. Consistently, epidermal thickening as well as the number of dermal mast cell was significantly lowered in ERW-bathing group. Defection of corneocytes under the scanning electron microscope was less observed in ERW-bathing group than in TW-bathing group. Further, the level of interleukin (IL)-1 $\beta$, tumor necrosis factor (TNF)- $\alpha$ and IL-12p70 in ERW group decreased whereas those of IL-10 increased. Collectively, our data indicate that ERW-bathing significantly reduces UVB-induced skin damage through influencing pro-/anti-inflammatory cytokine balance in hairless mice. This suggests that ERW-bathing has a positive effect on acute UVB-mediated skin disorders. This is the first report on bathing effects of ERW in UVB-induced skin injury.

Key words electrolyzed reduced water; UV-irradiation; bathing; skin; cytokine

Ultraviolet radiation (UV) is classified into 3 types: UVA $(320-400 \mathrm{~nm})$, UVB $(280-320 \mathrm{~nm})$ and UVC $(200-$ $280 \mathrm{~nm}$ ) which is blocked at stratospheric ozone layer. UVB can cause various skin disorders because the energy level of UV is inversely related to its wavelength and UVB has higher level than UVA. ${ }^{1)}$ UV irradiation can induce not only a variety of skin damages including erythema (sunburn inflammation), photosensitivity, and DNA damage resulting in immune suppression at short-term exposure but also photoaging and carcinoma at chronic exposure. ${ }^{2-6)} \mathrm{UV}$ irradiation also involves invisible changes of cell and gene level such as abnormal secretion of cytokines by keratinocytes, decreased Langerhans cells, increased mast cells and DNA damage relevant to pyrimidine dimers and photoproducts in skin. ${ }^{7-9)}$

Skin is biological defense barrier and a major target affected by UV-mediated damage directly or indirectly. Furthermore, skin is a major target of oxidative stress originated from the environmental agent such as X-ray, ozone, UV, air pollutants and industrial chemicals. ${ }^{10,11)}$ Reactive oxygen species (ROS) and reactive nitrogen species (RNS) generated by UV irradiation play a key role in the pathogenesis of UVinduced skin damage through both direct DNA damage and indirect ROS-mediated oxidative damage. ${ }^{12-14)}$ Furthermore, physiologic or pathologic dose of UVB irradiation affects local or systemic immunity through triggering cytokine production. Of note, cumulative UV-induced skin injury might break pro-/anti-inflammatory cytokine balance in skin barrier or even systemically. This immune dysfunction plus ROS would aggravate the function and structure skin barrier, consequently leading to photoaging. ${ }^{15)}$

In that context, many strategies were developed to prevent
ROS-induced cellular damage or immune dysfunction in skin barrier. For instance, wide array of antioxidant agents has been used in the form of foods, cosmetics and inhalant to reduce UV-induced skin damage. ${ }^{16-20)}$ However, convenient treatments or manipulations of UV-induced skin injury are still limited.

As a convenient, potential therapeutic candidate against UV-induced skin injury, we postulated that alkaline reduced water (ARW), kind of functional water, might be usable owing to antioxidant and anti-inflammatory effects, ${ }^{21,22)}$ and DNA protecting effect from oxidative damage by ROS. ${ }^{23)}$ ARW can be made by electrolysis of water in the cathode termed electrolyzed reduced water (ERW) or by reaction of water with mineral substances like magnesium. ARW is characterized by high alkalinity, extremely negative oxidation reduction potential (ORP) value and extremely high concentration of hydrogen. Many reports showed that ERW-drinking has been widely used for treatment and prevention of various medical diseases such as renal disease and diabetes. ${ }^{22-24)}$ Despite the beneficial, the approved effect of ARW drinking against some medical disease, long term intake of ARW is still a limitation to widely apply to clinical settings.

To overcome this drawback, we attempted new approach, ERW-bathing, as a potential strategy for treatment of acute UVB-mediated skin damage. Our hypothesis is that ERW bathing reduces UVB-induced skin damage through influencing pro-/anti-inflammatory cytokine balance in hairless mice. To verify this hypothesis, we investigated the effect of ERW-bathing through white blood cell (WBC) counting, glutathione peroxidase (GPx) activity, cytokine analysis, histological examination including epidermal thickness and 
dermal mast cell, and ultrastructural change of corneocytes.

\section{MATERIALS AND METHODS}

Preparation of ERW Electrolyzed reduced water (ERW) was obtained from electrolyzing apparatus (WYD35RY1, Ionia Inc., Korea). Electrolyzing apparatus equipped with a cathode platinum-coated titanium electrode $(0.9 \pm 0.1 \mathrm{~A}, 1-$ $2 \mathrm{l} / \mathrm{min}$ at $2 \mathrm{kgf} / \mathrm{min}$ ) produced ERW by following process: physical filtering of tap water, electrolysis and collecting ERW. ERW was adjusted to $\mathrm{pH} 10.27 \pm 0.3$ and oxidativereduction potential (ORP) $-494 \pm 20 \mathrm{mV}$. We used tap water (TW) for control group and it was $\mathrm{pH} 7.27 \pm 0.3$, ORP $+817 \pm 20 \mathrm{mV}$.

Animals Male hairless mice (6 weeks old, body weight $30 \pm 2 \mathrm{~g}$ ) were purchased from Orient Bio Inc. (Korea). They were housed in stainless steel cages in a controlled environment of $12 \mathrm{~h}$ light and dark cycle, humidity $55 \pm 5 \%$ and temperature $22 \pm 2{ }^{\circ} \mathrm{C}$. After 1 week of acclimation, the hairless mice were randomly divided into 4 groups $(n=11$, respectively): normal group with no treatment, UV-control group treated with UVB only, TW-bathing control group bathed in tap water after UV treatment and ERW-bathing group bathed in ERW after UV treatment. The animal use and care protocols for this animal experiment were approved by the IACUC (Institutional Animal Care and Use Committee), Wonju Campus, Yonsei University, Wonju, Gangwon, Korea.

UVB Irradiation and Bathing UVB fluorescent lamp (TL40W/12RS, Philips, Netherlands) emitting a continuous spectrum between $290-320 \mathrm{~nm}$ was used to induce skin damage. The intensity of UVB lamp was $0.15 \mathrm{~mW} / \mathrm{cm}^{2}$ and the total cumulative dose energy of UVB irradiated was $400 \pm 40 \mathrm{~mJ} / \mathrm{cm}^{2}$ (UV light meter YK-34UV, Lutron Electronics Inc., Taiwan). The lamp was mounted $30 \mathrm{~cm}$ above surface of the bathing bottle. The mice of TW-bathing and ERW-bathing groups were exposed to UVB lamp in the bathing bottle swimming freely simultaneously with bathing for $30 \mathrm{~min}$ a day during $3 \mathrm{~d}$, and then the mice were individually taken a bath for 30 min a day during $21 \mathrm{~d}$ without UVB irradiation. Two days after bathing without UVB irradiation, GPx activity and cytokine analysis (interleukin (IL)- $1 \beta$, IL-6, IL-10, IL-12p70, tumor necrosis factor (TNF)- $\alpha$ and interferon (IFN)- $\gamma$ ) was examined by Bio-Plex assay. The degree of injury on the back skin was scored by recording every other day for $21 \mathrm{~d}$. At the end of experiment, all the mice were sacrificed after anesthesia with $\mathrm{CO}_{2}$ gas for histological (epidermal thickness, and mast cell counting) and ultrastructural examination of corneocytes using scanning electron microscope. Water temperature for bathing was adjusted to $30 \pm 1{ }^{\circ} \mathrm{C}$. The mice of bathing groups were submerged up to $90 \%$ above of dorsal part into water chamber.

Scoring of Skin Injury From the next day after UVB irradiation, the degree of dorsal skin injury was observed at an interval of $2 \mathrm{~d}$ for $21 \mathrm{~d}$. The grades were divided into 8 steps according to the degree of skin. Score were presented as mean \pm S.E.M.

Hematological Examination Two days after UVB irradiation and bathing, mice were anesthetized with $\mathrm{CO}_{2}$, and blood samples were collected from the retro-orbital plexus to ethylenediaminetetraacetic acid (EDTA) tube. For hematological examination, the total WBC count, and numbers of neutrophils, lymphocytes, monocytes, eosinophils, and basophils were determined by an automatic blood analyzer (HEMAVET HV950 FS, Drew Scientific Inc., U.S.A.).

GPx (Glutathione Peroxidase) Activity Assay GPx activity in plasma was measured for $\mathrm{H}_{2} \mathrm{O}_{2}$ scavenging capacity by modified Cayman's GPx assay. ${ }^{25)}$ Oxidized glutathione, produced upon reduction of hydroperoxide by GPx, is recycled to its reduced state by glutathione reductase and reduced nicotinamide adenine dinucleotide phosphate (NADPH). The oxidation of NADPH to $\mathrm{NADP}^{+}$is accompanied by a decrease in absorbance at $340 \mathrm{~nm}$. Blood were collected from orbital plexus to EDTA tube after anesthesia with $\mathrm{CO}_{2}$ gas, centrifuged at $700-1000 \times \boldsymbol{g}$ for $10 \mathrm{~min}$ at $4{ }^{\circ} \mathrm{C}$ to get plasma. Plasma sample was stored at $-80^{\circ} \mathrm{C}$ before using. For background, $120 \mu \mathrm{l}$ assay buffer and $50 \mu \mathrm{l}$ co-substrate mixture were added to 3 wells. For positive control, $100 \mu \mathrm{l}$ assay buffer, and $50 \mu \mathrm{l}$ co-substrate mixture and $20 \mu \mathrm{l}$ diluted GPx (control) were added to 3 wells. For measurement of experimental samples, $100 \mu \mathrm{l}$ assay buffer, $50 \mu \mathrm{l}$ co-substrate mixture and $20 \mu \mathrm{l}$ plasma sample to 3 wells. Twenty microliters cumene hydroperoxide were added to all the wells to initiate the reactions. After shake the plate carefully for a few seconds to mix, we read the absorbance once every minute at $340 \mathrm{~nm}$ using a plate reader to obtain at least 5 time points. GPx activity was calculated by following formula. The reaction rate at $340 \mathrm{~nm}$ can be determined using the NADPH extinction coefficient of $0.00373 \mu \mathrm{M}^{-1}$. One unit is defined as the amount of enzyme that causes the oxidation of $1.0 \mathrm{~nm}$ of $\mathrm{NADPH}$ to $\mathrm{NADP}+$ per minute at $25^{\circ} \mathrm{C}$. The GPx activity was calculated using the following formula: GPx activity $(\mathrm{nM} / \mathrm{min} / \mathrm{ml})=\left(\Delta A_{340} / \mathrm{min}\right) /(0.00373 / \mu \mathrm{M}) \times(0.09 \mathrm{ml} / 0.02 \mathrm{ml}) \times$ sample.

Bio-Plex Cytokine Assay Serum samples were separated from blood by centrifuge of $14000 \mathrm{rpm}$ for $5 \mathrm{~min}$ and stored at $-70^{\circ} \mathrm{C}$ before use. For analysis of IL- $1 \beta$, IL$12 \mathrm{p} 70$, TNF- $\alpha$, IFN- $\gamma$, IL-10, and IL- 6 , anti-cytokine conjugated beads were diluted 1:25 per well using assay buffer, transferred into each plate well and washed on the wash platform 2 times. Samples, standards and controls respectively were mixed with anti-cytokine conjugated beads, and incubated for $30 \mathrm{~min}$. After washing, specific biotinylated detection anti-cytokines were added and incubated for $30 \mathrm{~min}$. Streptavidin-PE solution diluted with assay buffer A was added to each well, incubated for $10 \mathrm{~min}$, and washed 3 times with wash buffer A. One hundred microliters assay buffer A was added into each well containing the beads and read in multi-plex bead suspension array system (Bio-Plex 200, BIO-RAD ${ }^{\circledR}$, U.S.A.).

Histological Process for Light Microscopy For light microscopic observations, dorsal skins of hairless mice $\left(1 \mathrm{~cm}^{2}\right)$ were separated, fixed in $10 \%$ neutral buffered formalin $(0.1 \mathrm{M}$ phosphate buffer, $\mathrm{pH} 7.4)$, dehydrated through a graded ethanol series, cleared by xylene and embedded in paraffin wax (Polyscience, U.S.A.). The paraffin sections were cut $4 \mu \mathrm{m}$ thick by microtome (Reichert-Jung, U.S.A.), stained and observed under the light microscope (BA300, Motic Ltd., Taiwan).

Measurement of Epidermis Thickness To measure epidermis thickness, the sections were stained with hematoxylin-eosin (Sigma, U.S.A.). We measured 5 fields of epidermal layer per mouse using Motic Images Plus 2.0 pro- 
gram (Motic Ltd., Taiwan) at 400 magnifications of light microscope (BA300, Motic Ltd., Taiwan). Epidermal layer was defined from stratum basale to stratum granulosum because stratum corneum was severely separated and the space between stratum granulosum and stratum corneum irregularly widened by bathing.

Observation of Mast Cell The sections were stained with $0.5 \%$ toluidine blue solution $(\mathrm{pH} 4.5)$ for $20 \mathrm{~min}$ and washed. Water on the slide was removed by filter paper, dehydrated in acetone, cleared with xylene and mounted. From each section, five randomly picked areas were used to count mast cells in dermal layer without knowing the treatment group at 400 magnifications under a light microscope (BA300, Motic Ltd., Taiwan).

Scanning Electron Microscopy (SEM) For scanning electron microscopic observation, the specimens were fixed in $2.5 \%$ glutaraldehyde for $2 \mathrm{~h}$ at $4{ }^{\circ} \mathrm{C}$, washed in $0.1 \mathrm{M}$ phosphate buffer $(\mathrm{pH} 7.4)$ and then postfixed in $1 \%$ osmium tetroxide for $90 \mathrm{~min}$. The specimens were dehydrated in the graded ethanol, exchanged through isoamylacetate, and critical point dried. The specimens were coated with gold-palladium and then examined in a scanning electron microscope (TM-1000, Hitachi, Japan). All reagents were purchased from Polysciences, Inc. (U.S.A.).

Statistical Analysis All data are presented as mean士 S.E.M. Statistical analysis was performed using unpaired Student's $t$-test using Prism 5.0 (GraphPad Software, Inc., U.S.A.). A $p$ value of less than 0.05 was considered to be statistically significant.

\section{RESULTS}

Scoring of Skin Injury The next day after the last UVB-irradiation, back skin injury scores showed significant difference between experimental groups: UV-control group $<$ ERW-bathing group $(p<0.05 v s$. TW-bathing group) $<$ TWbathing group. However, on the third day, ERW-bathing group ( $p<0.001 v s$. TW-bathing group) showed the lowest score compared to the other groups: ERW-bathing group $(p<0.01$ vs. TW-bathing group $)<\mathrm{UV}$-control group $<\mathrm{TW}$ bathing group. Since then until the 21 st day, the score was generally decreased but not different at the end of experimental period. Collectively, UVB-mediated skin injury of ERW-bathing group revealed a significantly reduced damage in the early stage, which means protecting effect of ERWbathing against UVB radiation (Fig. 1). In addition, lumps were observed inside dorsal skin in 5 hairless mice of UVcontrol group, in 3 of TW-bathing group and 1 of ERWgroup.

WBC Counting The number of total WBC, neutrophils, and monocytes in the ERW-bathing group tends to decrease compared to TW-bathing group, while that of lymphocyte slightly increased. All the measured parameters had no significance between the groups (Table 1).

Glutathione Peroxidase (GPx) Activity $\mathrm{H}_{2} \mathrm{O}_{2}$ scavenging capacity in plasma was measured by the principles of glutathion peroxidase (GPx) activity decomposing $\mathrm{H}_{2} \mathrm{O}_{2}$ induced by UVB. GPx activity of ERW bathing group was higher than that of TW bathing although there was no significance (Fig. 2).

Bio-Plex Cytokine Assay Overall serum levels of IL-

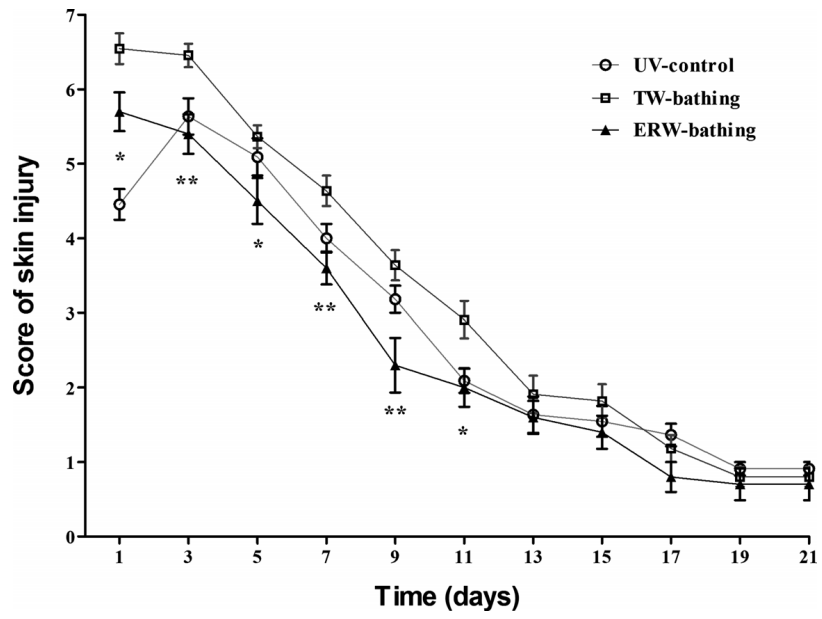

Fig. 1. Scoring of Skin Injury after UVB-Irradiation and Bathing Simultaneously

The grades were divided into 8 steps according to the degree of injury by naked eyes. UV-control: only UVB irradiated group, ERW-bathing: simultaneously UVB irradiation and ERW-bathing group, TW-bathing: simultaneously UVB irradiation and tap waterbathing group. Data were presented as mean \pm S.E.M. $* p<0.05 v$ s. TW-bathing group, $* * p<0.01$ vs. TW-bathing group.

Table 1. WBC Counting

\begin{tabular}{lcccc}
\hline \hline \multicolumn{1}{c}{ Groups } & Normal & UVB control & TW-bathing & ERW-bathing \\
\hline Total WBC & $4.64 \pm 0.95$ & $6.70 \pm 1.71$ & $7.41 \pm 3.21$ & $7.13 \pm 2.82$ \\
Neutrophil & $1.32 \pm 0.22$ & $3.00 \pm 1.10$ & $4.20 \pm 2.44$ & $3.89 \pm 2.18$ \\
Lymphocyte & $2.94 \pm 0.69$ & $3.22 \pm 0.69$ & $2.67 \pm 0.65$ & $2.80 \pm 0.66$ \\
Monocyte & $0.35 \pm 0.14$ & $0.43 \pm 0.13$ & $0.40 \pm 0.12$ & $0.39 \pm 0.12$ \\
Eosinophil & $0.02 \pm 0.01$ & $0.08 \pm 0.06$ & $0.04 \pm 0.03$ & $0.04 \pm 0.02$ \\
Basophil & $0.01 \pm 0.01$ & $0.01 \pm 0.01$ & $0.01 \pm 0.01$ & $0.01 \pm 0.01$ \\
\hline
\end{tabular}

Normal: no bathing and no UVB irradiation, UVB control: no bathing and UVB irradiation, TW-bathing and ERW-bathing: bathing TW and ERW, respectively and UVB irradiation. Data were presented as mean \pm S.E.M.

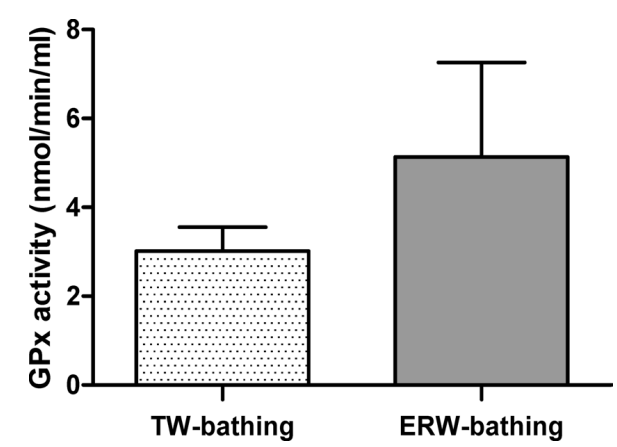

Fig. 2. Plasma GPx Activity of TW-Bathing and ERW-Bathing Group

Increased GPx activity of ERW-bathing group indicates increased $\mathrm{H}_{2} \mathrm{O}_{2}$ scavenging capacity. GPx activity of ERW-bathing group was higher than that of TW-bathing group. Data were presented as mean \pm S.E.M.

$12 \mathrm{p} 70$, INF- $\gamma$, IL-6, and IL-10 in bathing groups were higher than those of non-bathing groups. Of non-bathing groups, serum level of IL- $1 \beta$, INF- $\gamma$, TNF- $\alpha$, and IL-6 in UVB control group were higher than that of normal unbathed mice. Comparing cytokine levels between bathing groups, ERWbathing group showed lower level of proinflammatory cytokines (IL-1 $\beta$, TNF- $\alpha$, and IL-12p70) as well as higher level of anti-inflammatory cytokine (IL-10) than TW-bathing group (Fig. 3).

Histological Observation of Skin In the microscopic 

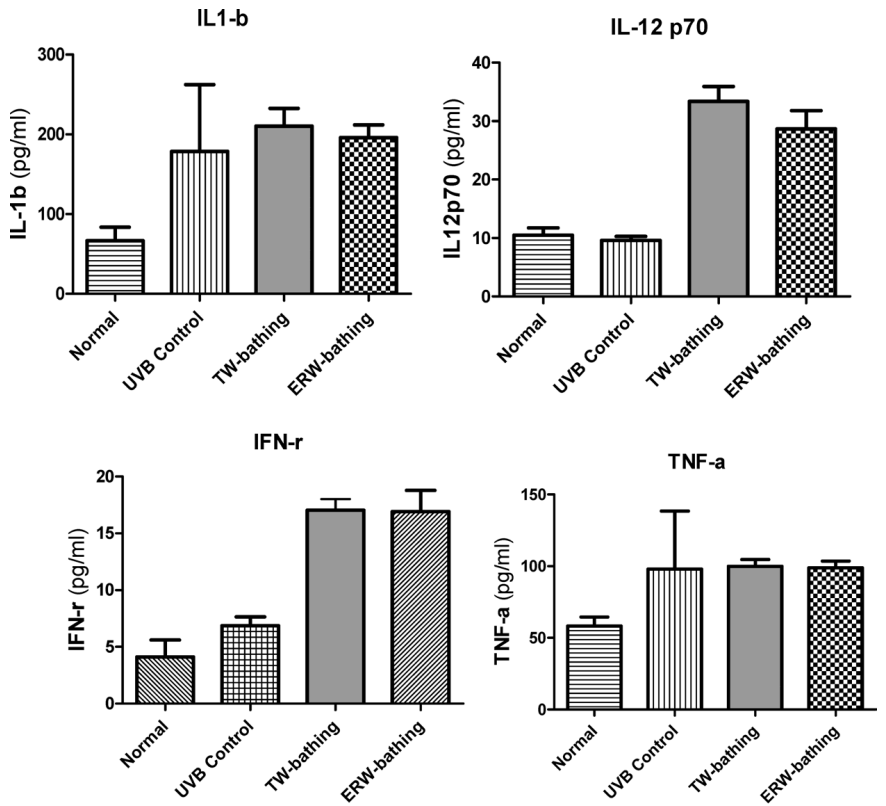

IL-6
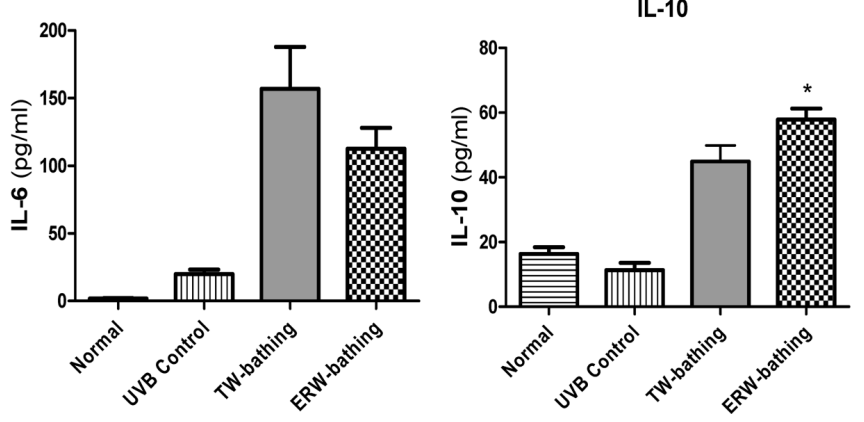

Fig. 3. Bio-Plex Cytokine Analysis

Normal and UVB-control groups were non bathe, while TW-bathing and ERWbathing groups were bathed in tap water and ERW, respectively, and UVB-control, TWbathing and ERW-bathing groups were exposed by UVB simultaneously. Data were presented as mean \pm S.E.M. $* p<0.05 v s$. TW-bathing group.

observation, dorsal skin of UV-control group showed excessive epidermis thickness accompanied by hyperplasia of stratum spinosum and stratum granulosum, severe inflammatory cell infiltration in dermis layer compared to the other groups. ERW-bathing group showed relatively decreased inflammatory cell infiltration compared to TW-bathing group (Fig. 4).

Comparing between bathing groups, epidermis thickness showed in the order of UV-control group $>$ TW-bathing group $>$ ERW-bathing group. In particular, epidermis thickness of ERW-bathing group was significantly decreased compared to that of TW-bathing group $(p<0.001)$ (Fig. 5).

In mast cell observation, the number of mast cell in dermis as well as the degree of degranulation in ERW-bathing group $(p<0.001)$ showed a significantly decreased compared to both the UV-control group and TW-bathing group (Fig. 6).

Ultrastructural Changes of Dorsal Skin Surface by SEM On the observation of dorsal skin surface by scanning electron microscope, UV-control group showed a lot of defective corneocytes which was severely distorted and separated each other. However, the degree of defection of both bathing groups was lower than UV-control group. Specifically in ERW-bathing group, the number of defected corneocytes and ultrastructural changes was markedly lower than
TW-bathing group (Fig. 7).

\section{DISCUSSION}

This study shows that ERW-bathing significantly reduces UVB-induced skin damage through influencing pro-/anti-inflammatory cytokine balance in hairless mice. This evidence stems from histological examination such as scoring of skin injury, the epidermal thickness, dermal mast cells and ultrastructural change of corneocytes, as well as GPx activity and cytokine analyses.

To prove our hypothesis, we designed in vivo skin injuryhealing model: the first step is UVB irradiation into the dorsal skin in hairless mice followed by simultaneous bathing in ERW and TW and next, is to bathe mice in specialized chamber for $30 \mathrm{~min}$ a day during $21 \mathrm{~d}$. Prior to histological study, first we examined the clinical score of UVB-injured skin in mice. We found that after $72 \mathrm{~h}$ of UV irradiation with bathing, the scores of UVB-inflicted injuries were significantly lower in ERW-bathing group than TW-bathing group, and were significantly different over the next $12 \mathrm{~d}$.

Next to obtaining the histological evidence, we sequentially measured the different, reliable parameters of skin injury: the epidermal thickness, the numbers of inflammatory cells and dermal mast cell, and ultrastructural change of corneocytes.

First, as we anticipated, the epidermis thickness of ERWbathing group was significantly decreased compared to that of the normal control mice (Fig. 5). In UVB-irradiated skin, the epidermis reactive hyperplasia might occur through ROS, particularly superoxide and hydrogen peroxide. Thus, the resultant prooxidant state in skin barrier accelerates epidermal hyperplasia as well as corneal thickening and melanization. Therefore, the lesser epithelial hyperplasia in ERW-bathing group might reflect more effective skin protection of ERW from UV-induced oxidative stress. Higher GPx activity of ERW bathing group might support such a skin protection. However, further skin GPx activity would be better correlated with.

Next, the examination of dermal mast cells with WBC counting showed that ERW-bathing significantly reduced the number of dermal mast cells and mast cell degranulation (Fig. 6), and decreased the number of total WBC and neutrophils in blood (Table 1) compared to that of TW-bathing. Mast cell plays an essential role in the induction and the persistence of inflammatory skin reactions. ${ }^{25,26)}$ High dose UVB exposure can directly or indirectly (oxidative stress) damage the cell membrane of mast cells, release of large number of biologically active substances such as vasoactive substances, chemokines, enzymes and structural proteins, and result in immunosuppression. ${ }^{27,28)}$ Given these, these cellular data might be the direct evidence that ERW might exert antiinflammatory effects in skin barrier.

Taken together, ERW might affect the mast cell function, stabilize the skin inflammation persisted by mast cells and influence immunological homeostasis. To further delineate the plausible anti-inflammatory effect of ERW bathing, we attempted serum cytokine profiling to gauge pro-/anti-inflammatory cytokine balance. In ERW-bathing group, lower level of pro-inflammatory cytokines (IL- $1 \beta$, TNF- $\alpha$, and IL$12 p 70)$ as well as higher level of anti-inflammatory cytokine 

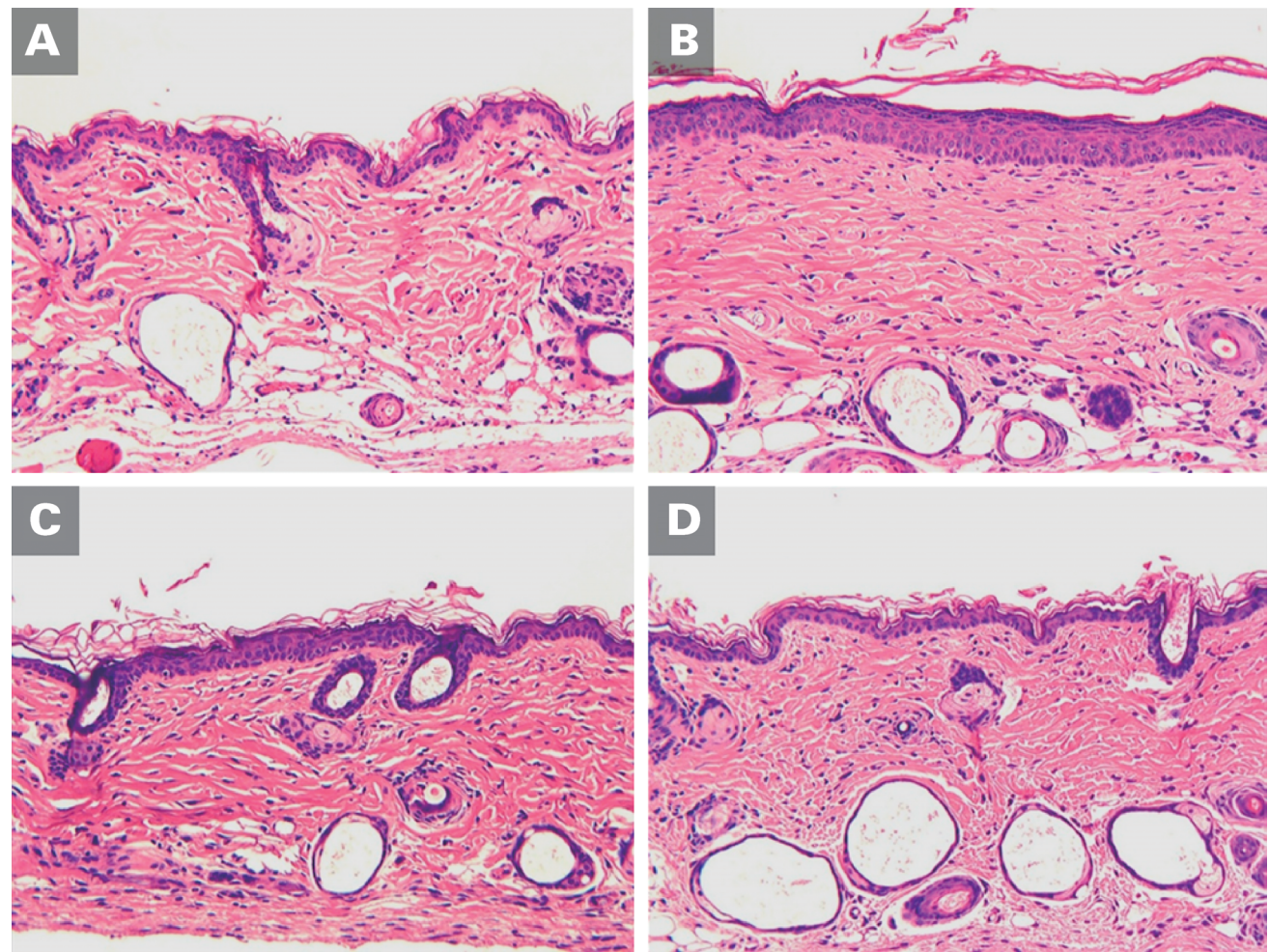

Fig. 4. Micrograph for Observation of Inflammatory Cells in Dermal Layer of Skin

(A) Normal group, (B) UVB-control group, (C) TW-bathing group, (D) ERW-bathing group, hematoxyline-eosin stain, 200 magnifications

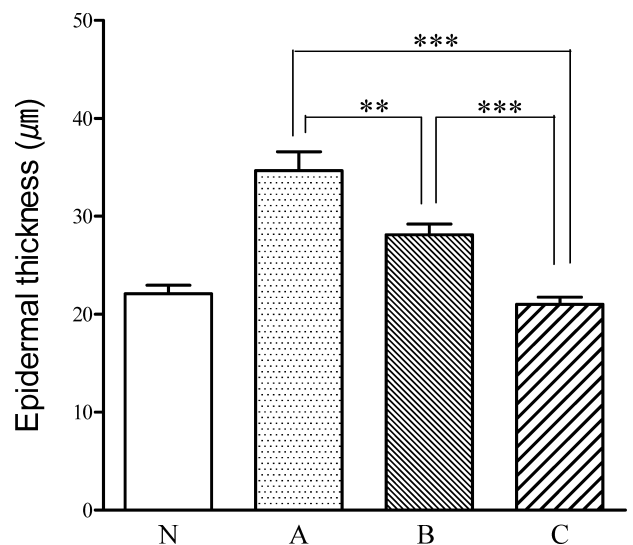

Fig. 5. Epidermal Thickness between Stratum Basale to Stratum Granulosum in Skin

N: Normal group, A: UVB-control group, B: TW-bathing group, C: ERW-bathing group. Data were presented as mean \pm S.E.M. $* * p<0.01, * * * p<0.001$.

(IL-10) might be compatible with cellular- and histological results. Cumulative evidence showed that UVB leads to a shift from the production of pro-inflammatory cytokines such as IL-1, IL-12, IL-8, TNF- $\alpha$ and IFN- $\gamma$ to the production of anti-inflammatory cytokines such as IL-4, IL-10 and IL$13 .^{29-31)}$ Breakdown of pro- and anti-inflammatory cytokines leads to various disease such as atopic dermatitis, rheumatoid arthritis and psoriasis. Based on cytokine profiling, ERWbathing definitely restored such a pro- and anti-inflammatory cytokine imbalance evoked by UV irradiation. UVB irradiation on the skin would induce alteration of antigen presenting cells including Langerhans cells and imbalance of a cell-mediated immune response. Interleukin 10 is known as an important immunoregulatory cytokine to downregulate inflam-

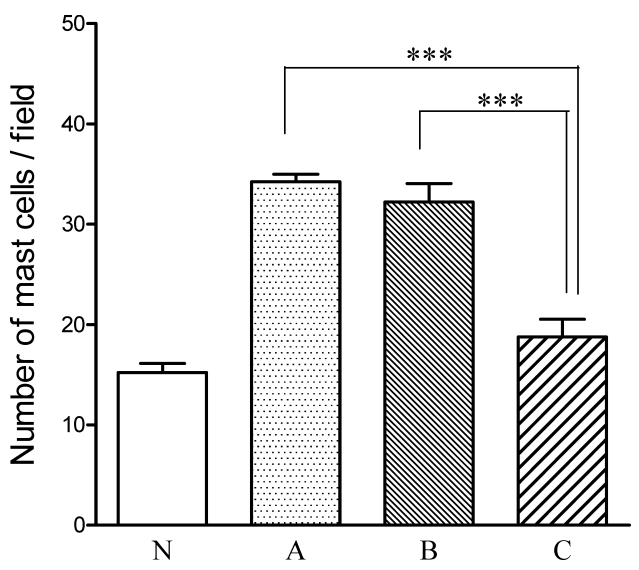

Fig. 6. The Number of Mast Cells in Dermal Layer

Mast cells were counted in five fields per under the light microscope of 400 magnification. N: normal group, A: UVB-control group, B: TW-bathing group, C: ERWbathing group. Data were presented as mean \pm S.E.M. $* * * p<0.001$.

matory responses and the regulate differentiation and proliferation of several immune cells such as T cells, B cell, natural killer (NK) cells mast cells and granulocytes. ${ }^{32,33)}$ In that context, this immunomodulatory effect of ERW-bathing might justify the clinical therapeutic usage for skin inflammatory disorders characterized by cytokine imbanace. ${ }^{34)}$

Surprisingly, ERW bathing reduced the number of mast cell in dermis to the level of normal control. Last, to elucidate ultrastructure of ERW bathed skin, we performed the scanning electron microscope on the number of defected corneocytes. Specifically, in ERW-bathing group, the number of defected corneocytes and ultrastructural changes was markedly lower than TW-bathing group. This result confers the ultrastructural clue to uncover the mechanism of ERW 

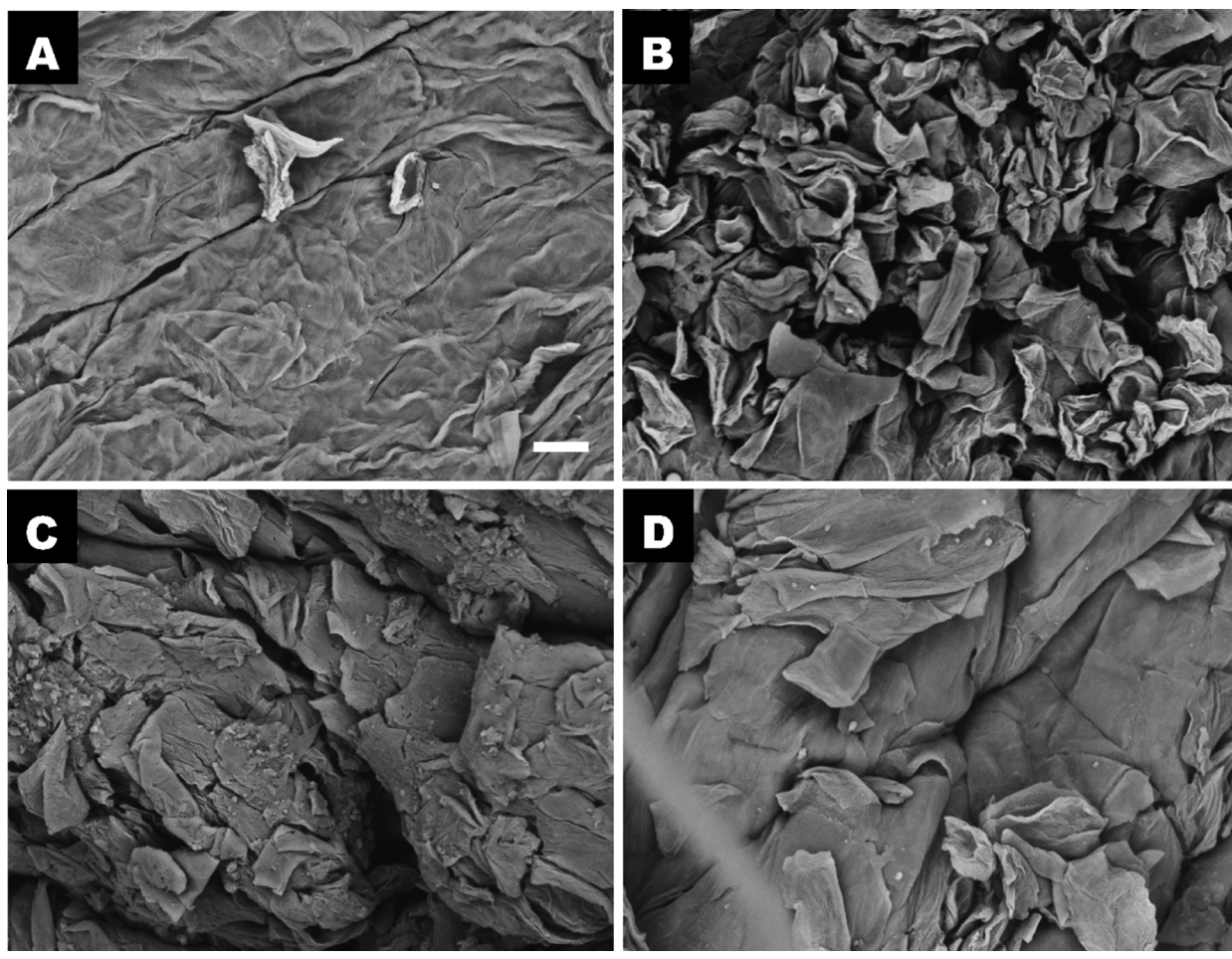

Fig. 7. Scanning Electron Micrographs of the Skin Surface of Dorsal Part in Hairless Mouse (Scale Bar=10 $\mu \mathrm{m}$ )

(A) Normal group, (B) UVB-control group, (C) TW-bathing group, (D) ERW-bathing group.

bathing. This is the first report on beneficial bathing effects of ERW on the UVB-induced skin injury.

However, our study had some limitations: the lack of detailed molecular functional mechanism for the effect of ERW bathing. For instance, further study on time-course and dose-effect relationship would be warranted since different doses of UVR may give rise to different manifestations at different time intervals in the cellular oxidative stress. ${ }^{35-37)}$ Our preliminary human study revealed that ERW-like hot spring bathing group revealed significantly lower ROS concentration in blood than TW-bathing group at the same water temperature and bathing time (data not shown). This experiment suggests that current results from ERW-bathing might be closely associated with ROS scavenging activity of ERW. $^{38)}$ In line, GPx activity of this study showed that ERWbathing might scavenge ROS although there is no significant. Likewise, our histological and immunological evidences of ERW bathing study strongly suggest that the ROS scavenging effect of ERW. Current evidences showed that hydrogen in ERW might play key roles for antioxidant effect of ERW.

Ohsawa et al. ${ }^{39)}$ demonstrated that hydrogen molecules rapidly diffuse across cell membranes followed by selectively scavenged hydroxyl radicals in vitro. Fukuda et al. ${ }^{40)}$ demonstrated hydrogen inhalation had therapeutic potential on hepatic ischemia/reperfusion injury in vivo through reduction of oxidative stress. In this study, hydrogen dissolved in ERW might be a plausible molecule to explain protective effect of ERW against acute UVB-mediated skin injury. Another merit of our study is to provide the rationale that ERW bathing might be a convenient, potential therapeutic candidate against UV-induced skin injury.

This hold the clinically or industrially important implication: clinical application of ERW bathing for the control of skin barrier disease, the possible application of ERW-like hot spring. Further study to uncover the molecular mechanism of ERW bathing is underway.

Collectively, our data indicate that ERW-bathing significantly reduces UVB-induced skin damage through influencing pro-/anti-inflammatory cytokine balance in hairless mice. This suggests that ERW-bathing has a positive effect on acute UVB-mediated skin disorders. This is the first report on bathing effects of ERW in UVB-induced skin injury.

Acknowledgements This work was supported by the National Research of Korea Grant funded by the Korean Government (NRF-2010-413-B00024).

\section{REFERENCES}

1) Clydesdale G. J., Dandie G. W., Muller H. K., Immunol. Cell Biol., 79 , $547-568$ (2001).

2) Trouba K. J., Hamadeh H. K., Amin R. P., Germolec D. R., Antioxid. Redox Signal., 4, 665-673 (2002).

3) Hennessy A., Oh C., Rees J., Diffey B., Photodermatol. Photoimmunol. Photomed., 21, 229-233 (2005).

4) Matsumura Y., Ananthaswamy H. N., Toxicol. Appl. Pharmacol., 195, 298-308 (2004).

5) Imokawa G., Arch. Dermatol. Res., 1, S7-S20 (2008).

6) Kwon O. S., Yoo H. G., Han J. H., Lee S. R., Chung J. H., Eun H. C., Arch. Dermatol. Res., 300, 47-52 (2008).

7) Engel A., Johnson M. L., Haynes S. G., Arch. Dermatol., 124, 72-79 (1988).

8) Situm M., Buljan M., Cavka V., Bulat V., Krolo I., Mihic L. L., Coll. Antropol., 34 (Suppl. 2), 9-13 (2010).

9) Dumay O., Karam A., Vian L., Moyal D., Hourseau C., Stoebner P., Peyron J. L., Meynadier J., Cano J. P., Meunier L., Br. J. Dermatol., 144, 1161-1168 (2001)

10) Halliwell B., Cross C. E., Environ. Health Perspect., 10, 5-12 (2000).

11) McArdle F., Rhodes L. E., Parslew R., Jack C. I., Friedmann P. S., 
Jackson M. J., Free Radic. Biol. Med., 33, 1355-1362 (2002).

12) Larsson P., Ollinger K., Rosdahl I., Br. J. Dermatol., 155, 292-300 (2006).

13) Van Laethem A., Nys K., Van Kelst S., Claerhout S., Ichijo H., Vandenheede J. R., Garmyn M., Agostinis P., Free Radic. Biol. Med., 41, 1361-1371 (2006).

14) Kochevar I. E., Lambert C. R., Lynch M. C., Tedesco A. C., Biochim. Biophys. Acta, 1280, 223-230 (1996).

15) Leavy O., Nat. Rev. Immunol., 7, 322-323 (2007).

16) Jin G. H., Liu Y., Jin S. Z., Liu X. D., Liu S. Z., Radiat. Environ. Biophys., 46, 61-68 (2007).

17) Lopez-Torres M., Thiele J. J., Shindo Y., Han D., Packer L., Br. J. Dermatol., 138, 207-215 (1998).

18) Katiyar S. K., Afaq F., Perez A., Mukhtar H., Carcinogenesis, 22, 287-294 (2001)

19) Fuchs J., Kern H., Free Radic. Biol. Med., 25, 1006-1012 (1998).

20) F'quyer S., Afaq F., Mukhtar H., Photodermatol. Photoimmunol. Photomed., 19, 56-72 (2003).

21) Hanaoka K., Sun D., Lawrence R., Kamitani Y., Fernandes G., Biophys. Chem., 107, 71-82 (2004).

22) Huang K. C., Yang C. C., Lee K. T., Chien C. T., Kidney Int., 64, $704-714$ (2003).

23) Shirahata S., Kabayama S., Nakano M., Miura T., Kusumoto K., Gotoh M., Hayashi H., Otsubo K., Morisawa S., Katakura Y., Biochem. Biophys. Res. Commun., 234, 269-274 (1997).

24) Jin D., Ryu S. H., Kim H. W., Yang E. J., Lim S. J., Ryang Y. S., Chung C. H., Park S. K., Lee K. J., Biosci. Biotechnol. Biochem., 70, 31-37 (2006).

25) Paglia E. E., Valentine W. N., J. Lab. Clin. Med., 70, 158-169 (1967).
26) Arock M., Zuany-Amorim C., Singer M., Benhamou M., Pretolani M., Eur. J. Immunol., 26, 166-170 (1996).

27) Hart P. H., Grimbaldeston M. A., Swift G. J., Jaksic A., Noonan F. P., Finlay-Jones J. J., J. Exp. Med., 187, 2045-2053 (1998).

28) Van Nguyen H., Di Girolamo N., Jackson N., Hampartzoumian T., Bullpitt P., Tedla N., Wakefield D., Scand. J. Rheumatol., 40, 197-204 (2011).

29) Scordi I. A., Vincek V., Photodermatol. Photoimmunol. Photomed., 16, 67-73 (2000).

30) Meeran S. M., Punathil T., Katiyar S. K., J. Invest. Dermatol., 128, 2716-2727 (2008)

31) Kupper T. S., Chua A. O., Flood P., McGuire J., Gubler U., J. Clin. Invest., 80, 430-436 (1987).

32) Asadullah K., Sterry W., Volk H. D., Pharmacol. Rev., 55, 241-269 (2003).

33) al-Janadi M., al-Dalaan A., al-Balla S., al-Humaidi M., Raziuddin S., J. Clin. Immunol., 16, 198-207 (1996).

34) Asadullah K., Sabat R., Wiese A., Döcke W. D., Volk H. D., Sterry W., Arch. Dermatol. Res., 291, 628-636 (1999).

35) Liu S. Z., Crit. Rev. Toxicol., 33, 431-441 (2003).

36) Danno K., Horio T., Br. J. Dermatol., 107, 423 - 428 (1982).

37) Shindo Y., Hashimoto T., J. Dermatol. Sci., 17, 140-150 (1998).

38) Yoon Y. S., Kim K. D., Kim N. I., Chi Y. H., Kim H. J., Lee K. J., Korean J. Waters, 1, 7-12 (2010).

39) Ohsawa I., Ishikawa M., Takahashi K., Watanabe M., Nishimaki K., Yamagata K., Katsura K., Katayama Y., Asoh S., Ohta S., Nat. Med., 13, 688-694 (2007).

40) Fukuda K., Asoh S., Ishikawa M., Yamamoto Y., Ohsawa I., Ohta S., Biochem. Biophys. Res. Commun., 361, 670—674 (2007). 